\title{
Catabolism of Branched-Chain Amino Acids in Heart Failure: Insights from Genetic Models
}

\author{
Haipeng Sun $\cdot$ Gang Lu $\cdot$ Shuxun Ren $\cdot$ \\ Jaunian Chen $\cdot$ Yibin Wang
}

Received: 29 November 2010/Accepted: 3 December 2010/Published online: 7 January 2011

(C) The Author(s) 2010. This article is published with open access at Springerlink.com

\begin{abstract}
Genetic defects in amino acid metabolism are major causes of newborn diseases that often lead to abnormal development and function of the central nervous system. Their direct impact on cardiac development and function has rarely been investigated. Recently, the authors have established that a mitochondrial targeted 2C-type ser/ thr protein phosphatase, $\mathrm{PP} 2 \mathrm{Cm}$, is the endogenous phosphatase of the branched-chain alpha keto acid-dehydrogenase complex (BCKD) and functions as a key regulator in branched-chain amino acid catabolism and homeostasis. Genetic inactivation of PP2Cm in mice leads to significant elevation in plasma concentrations of branched-chain amino acids and branched-chain keto acids at levels similar to those associated with intermediate mild forms of maple syrup urine disease. In addition to neuronal tissues, PP2Cm is highly expressed in cardiac muscle, and its expression is diminished in a heart under pathologic stresses. Whereas phenotypic features of heart failure are seen in $\mathrm{PP} 2 \mathrm{Cm}$ deficient zebra fish embryos, cardiac function in PP2Cmnull mice is compromised at a young age and deteriorates faster by mechanical overload. These observations suggest that the catabolism of branched-chain amino acids also has physiologic significance in maintaining normal cardiac function. Defects in PP2Cm-mediated catabolism of branched-chain amino acids can be a potential novel mechanism not only for maple syrup urine disease but also for congenital heart diseases and heart failure.
\end{abstract}

H. Sun · G. Lu · S. Ren · J. Chen · Y. Wang $(\bowtie)$

Division of Molecular Medicine,

Departments of Anesthesiology, Medicine and Physiology,

Molecular Biology Institute, Cardiovascular Research

Laboratories, David Geffen School of Medicine, CSH, Room BH

569, 650 Charles E. Young Drive, Los Angeles, CA 90095, USA

e-mail: yibinwang@mednet.ucla.edu
Keywords Amino acid metabolism · Branched-chain amino acid catabolism - Branched-chain alpha keto acid-dehydrogenase complex $\cdot \mathrm{PP} 2 \mathrm{Cm}$

\section{Amino Acid Metabolism and Genetic Disorders}

Amino acids are key nutrient molecules essential for cell growth, survival, and normal function. In addition to providing building blocks for protein synthesis, many amino acids are an essential ingredient for biosynthesis of other molecules as the sources of nitrogen and carbon [8]. Some amino acids, including branched-chain amino acids, also have been shown to possess a potent signaling function to regulate global growth and metabolism $[2,13,14,17,18$, $22,23]$. Therefore, the impact of amino acid metabolism on embryonic development and human congenital diseases has long been recognized.

Maple syrup urine disease, one of the most common genetic disorders caused by defects in amino acid metabolism, is a target of mandatory newborn screening in most of the United States and throughout the world $[5,10,20]$. The clinical manifestations of classical maple syrup urine disease are mostly neuropathologic, including seizure and mental retardation [7]. However, recent studies from animal models of maple syrup urine disease raise concerns about the potential adverse impact of branched-chain amino acid metabolic defects on cardiac development and function. These concerns are the focus of the discussion in this review.

\section{Catabolism of Branched-Chain Amino Acids}

Branched-chain amino acids (BCAAs) including leucine, isoleucine, and valine are essential amino acids that must 
be acquired from external food. In addition to their abundant presence in protein peptides as key hydrophobic building blocks, BCAAs also serve as significant sources for biosynthesis of sterol, keto bodies, and glucose [3]. Among the BCAAs, particularly leucine has potent signaling activity to promote protein synthesis, cellular metabolism, and cell growth in a mammalian target of rapamycin (mTOR)-dependent manner [4, 15, 19].

Although BCAAs are necessary for normal growth and function at cellular and organism levels, an excess amount of free BCAAs also can be pathologic. A high plasma level of BCAAs is the benchmark and cause of maple syrup urine disease, a potentially life-threatening disorder affecting 1 of 180,000 newborn babies on the average in the general population, with a much higher prevalence among certain Amish, Mennonite, and Jewish communities $[5,20]$.

Because BCAAs are essential amino acids with no biosynthesis pathways in animal cells, their homeostasis is determined largely by catabolic activities in a number of organs, particularly the liver [9]. The first step in the catabolism of BCAAs is carried out in brain, muscle, and many nonhepatic tissues by the branched-chain aminotransferase (BCAT) to convert BCAAs into branched-chain alpha keto acids (BCKAs) [9]. The BCKAs are decarboxylated by the branched-chain alpha keto acid dehydrogenase (BCKD) complex in the liver as well as other tissues and eventually degraded into acetyl-coenzyme A (CoA) or succinyl-CoA to fuel the TCA cycle. As BCKD mediates this rate-limiting step in BCAA catabolism, its activity dictates the steadystate levels of BCAA and BCKA. It thus is a target of multiple regulatory mechanisms, including cyclic adenosine monophosphate (cAMP)-mediated induction of RNA transcription and phosphorylation-mediated inhibition/activation of its enzymatic activity.

The BCKD complex is genetically linked with the pyruvate dehydrogenase complex (PDH) because they share extensive homology in their subunit composition and regulation. Like PDH, BCKD holoenzyme contains 24 copies of catalytic subunits E2/E3 and an equal number of regulatory subunits $\mathrm{E} 1 \alpha$ and $\mathrm{E} 1 \beta$. At low BCAA levels, $\mathrm{E} 1 \alpha$ is hyperphosphorylated by BCKD kinase, leading to lower BCKD activity and reduced loss of BCAA. At a high BCAA level, E1 $\alpha$ is dephosphorylated by BCKD phosphatase, leading to induced BCKD activity and the removal of excess BCAA. Therefore, BCKD phosphorylation/ dephosphorylation is critical to BCAA homeostasis [9].

\section{Molecular Identification of BCKD Phosphatase}

Based on the importance of BCKD phosphorylation in its regulation, BCKD phosphatase has been well established as a key regulator in BCAA catabolism. However, its molecular identity has remained elusive for decades. Recently, through genome scanning, we discovered a mitochondrial targeted 2C-type ser/thr protein phosphatase that we named PP2C in mitochondria (PP2Cm) [11].

Through extensive proteomic and biochemical analysis, we established that $\mathrm{PP} 2 \mathrm{Cm}$ is the missing BCKD phosphatase responsible for BCAA-induced dephosphorylation and activation of BCKD activity [12]. This conclusion is based on the following lines of evidence: (1) BCKD subunits, including E2 and $\mathrm{E} 1 \alpha$, are specifically associated with $\mathrm{PP} 2 \mathrm{Cm}$ in vitro and in vivo; (2) $\mathrm{PP} 2 \mathrm{Cm}$ expression effectively dephosphorylates E1 $\alpha$ Ser-293 phosphorylation, a key residue in BCKD enzymatic regulation; (3) PP2Cmdeficient cells have elevated basal $\mathrm{E} 1 \alpha$ phosphorylation, which remains high at BCKA treatment; (4) PP2Cm-deficient mice have a significantly higher plasma level of BCAA and BCKA with impaired BCAA clearance after a high dose of BCAA ingestion; and (5) PP2Cm-deficient newborn mice have significantly higher mortality under a high-protein diet challenge.

All these data establish that $\mathrm{PP} 2 \mathrm{Cm}$ is the endogenous BCKD phosphatase with an essential function in BCAA catabolism. The analysis with $\mathrm{PP} 2 \mathrm{Cm} \mathrm{KO}$ mice indicates that a defect in $\mathrm{PP} 2 \mathrm{Cm}$ is a potential novel mechanism for the intermediate/inducible forms of maple syrup urine disease.

\section{PP2Cm in Cardiac Regulation}

The discovery of $\mathrm{PP} 2 \mathrm{Cm}$ as the $\mathrm{BCKD}$ phosphatase and a key regulator in BCAA metabolism as well as the establishment of PP2Cm-deficient zebra fish and mouse models offers an opportunity to investigate the impact of BCAA regulation in intact animals. In both zebra fish embryos and adult mice, $\mathrm{PP} 2 \mathrm{Cm}$ is highly expressed in both cardiac muscle and the central nervous system (Figs. 1, 2). Its expression in the heart is dynamically regulated by stress, as measured by both mRNA and protein levels, with significantly reduced levels in hypertrophic and failing hearts [11].

It is not clear whether loss of PP2Cm is a contributing factor to cardiac pathology or simply a phenomenon associated with the diseased hearts. To investigate this question, we analyzed the cardiac performance in PP2Cm-deficient zebra fish and mice. Using morpholingoes specifically targeted to zPP2Cm translation initiation codon (ATG-MO), we demonstrated that. PP2Cm-deficient fish embryos displayed a dose-dependent loss of cardiac contractility and that most of them did not survive beyond the early embryonic stage [11]. Accelerated heart failure developed in PP2Cmdeficient mice after mechanical overload induced by transaortic constriction (Fig. 3). 

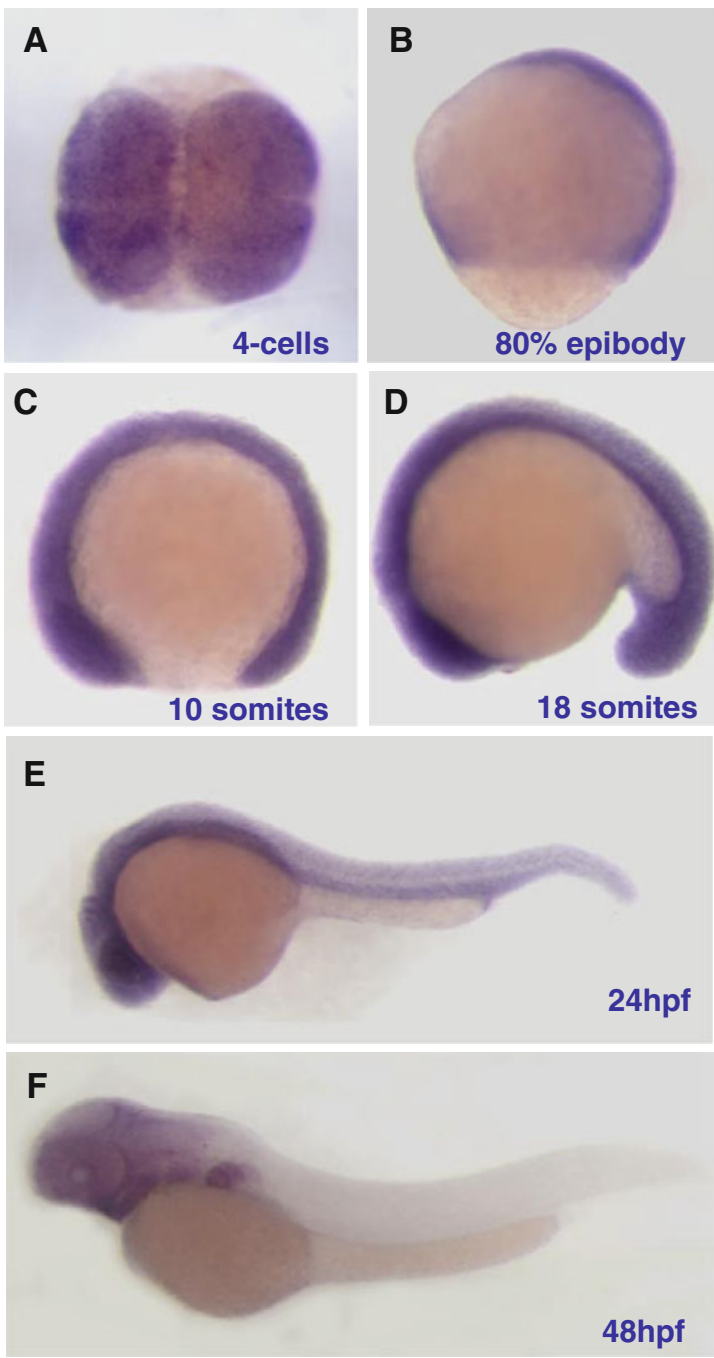

Fig. 1 Expression pattern of $\mathrm{PP} 2 \mathrm{C}$ in mitochondria (PP2Cm) of developing zebra fish embryos. a-f In situ hybridization signal of fPP2Cm mRNA in developing zebra fish embryos at a specific stage as indicated (hpf: hour postfertilization). g Transgene construct of

These evidences suggest that loss of $\mathrm{PP} 2 \mathrm{Cm}$ is not a mere consequence but rather a significant contributor to the pathogenesis of heart failure. These studies for the first time implicated BCAA catabolism also as an important aspect of cardiac pathophysiology and showed that defects in BCAA homeostasis can have a significant adverse impact on cardiac function and disease progression.

The underlying mechanisms for the clinical manifestations of maple syrup urine disease still are not well established, and the adverse impact on glutamine transport and the induction of reactive-oxygen species (ROS) have been implicated [1, 6, 24]. In our own studies with cardiac tissue or cultured cardiomyocytes, we also explored a number of these possible mechanisms.

1. We examined the cytotoxic effect of the accumulated BCAA/BCKA on cadiomyocyte survival, demonstrating

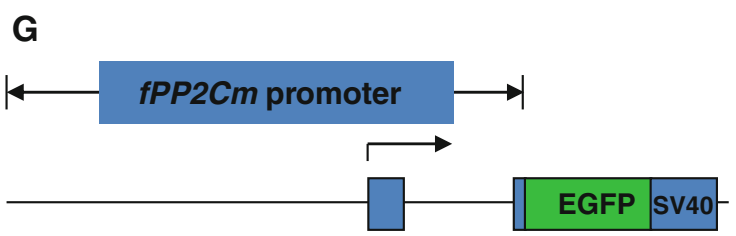

\section{H}
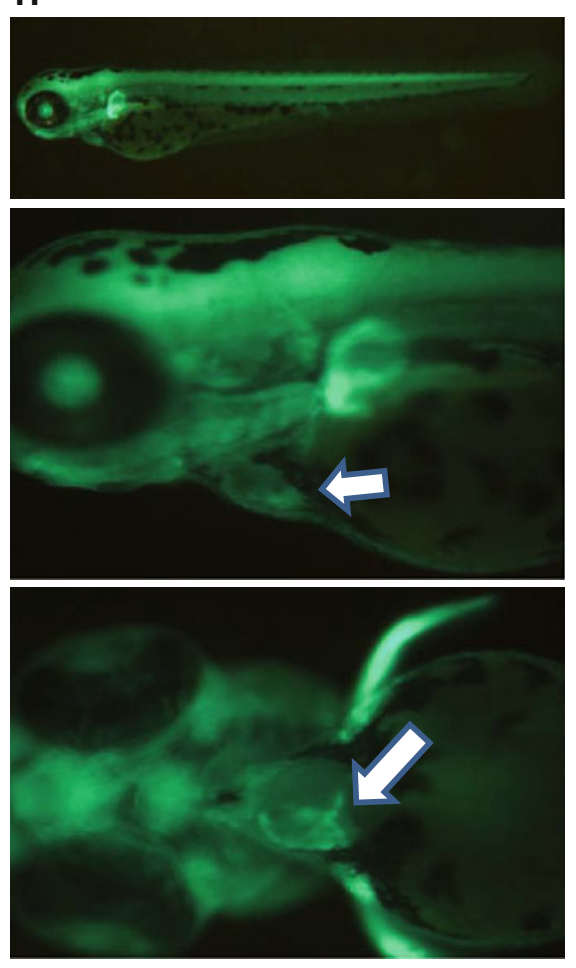

fPP2Cm promoter driving GFP expression. h GFP signal observed under ultraviolet illumination in transgenic zebrafish at $72 \mathrm{hpf}$ stage under different magnifications. Arrows indicate heart tube

that PP2Cm-deficient mice have a significantly elevated ROS level and are more susceptible to calcium-induced permeability transition pore opening in mitochondria [11]. Whether and how this effect is caused by accumulated BCAA or BCKA remains to be investigated further. However, a clear implication of the observed cytotoxicity is more cell death. Indeed, we observed that PP2Cm deficiency can lead to more apoptotic cell death in both developing embryos and cultured myocytes.

2. Metabolic effects of accumulated BCAA/BCKA can be pathologic in the heart. It is known that BCAA/ BCKA can inhibit pyruvate and fatty acid transport and utilization [16, 19]. Because cardiac tissue has a constant high demand for pyruvate and fatty acid as its main fuel source, chronically elevated BCAA/BCKA can potentially block normal bioenergenic homeostasis 
Fig. 2 Loss of $\mathrm{fPP} 2 \mathrm{Cm}$ expression leads to heart failure in zebra fish embryo. a Video frames of $\mathrm{cmlc}$-GFP transgenic fish embryo recorded under treatment with different concentrations of $\mathrm{PP} 2 \mathrm{Cm}$ morpholigo. b M-mode image of zebra fish hearts from linescanning analysis as shown in A as well as heart rate (HR) and percentile fractional shortening from $\mathrm{Lu}$ et al. [11] with permission ultraviolet illumination after (FS\%) measurements. Adapted

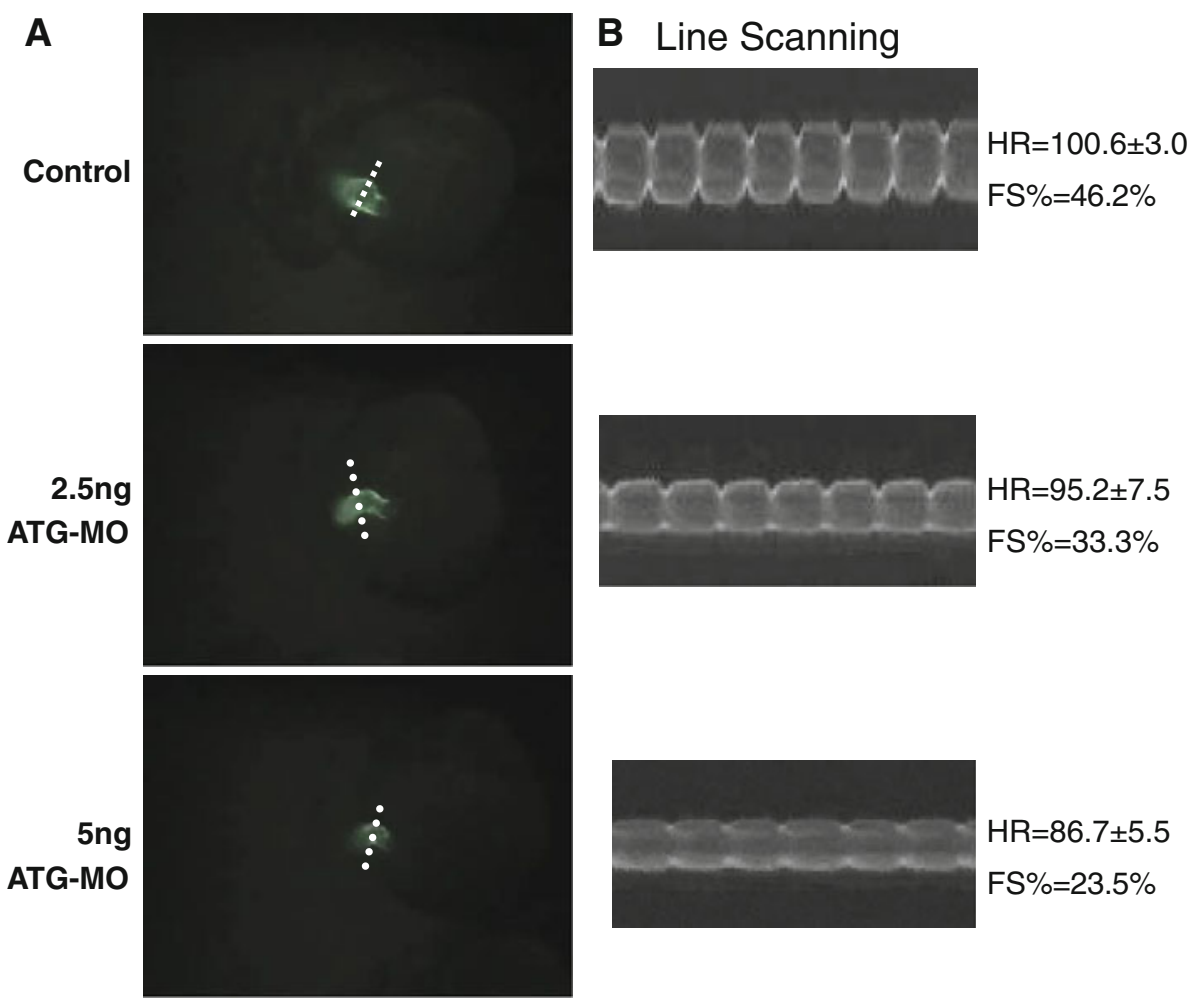

A

B Wildtype

Fig. 3 Expression and function of $\mathrm{PP} 2 \mathrm{Cm}$ in the heart.

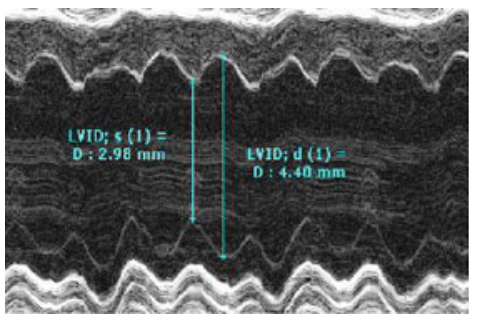

HR: 545, \%EF: 60.59, \%FS: 32.17

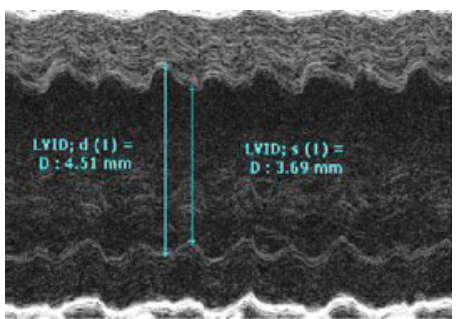

HR: 545, \%EF: 37.85, \%FS: 18.18

PP2Cm KO

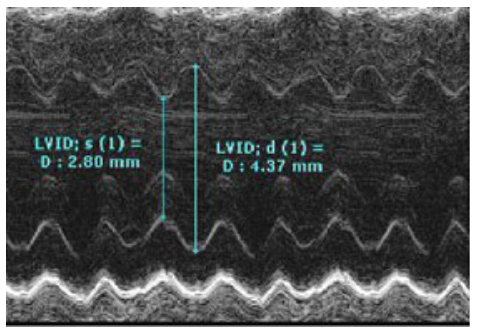

HR: 533, \%EF: 65.69, \%FS:35.88 Pre-TAC

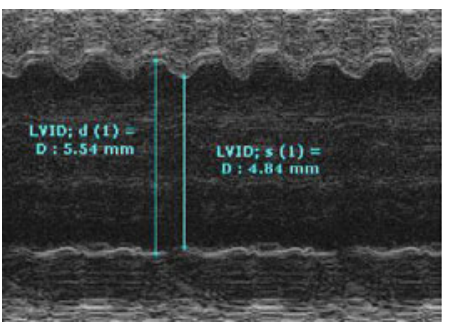

HR: 545, \%EF: 26.61, \%FS: 12.50 Post-TAC in the heart, leading to contractile dysfunction and accelerated heart failure under increased mechanical load or aging. Although we observed significant changes in a number of metabolic genes in PP2Cmdeficient hearts (Lu et al., unpublished results), a direct analysis of cardiac metabolic status and energy consumption is needed to support this hypothesis.
3. Our findings showed that $\mathrm{PP} 2 \mathrm{Cm}$ mediated direct modification of mitochondrial function in cellular bioenergenics and survival. Although BCKD is the only substrate identified for $\mathrm{PP} 2 \mathrm{Cm}$ to date, it is possible that other uncharacterized substrates in mitochondria contribute to PP2Cm-mediated signaling. This is supported by our observation that the isolated 
PP2Cm-deficient mitochondria have abnormal sensitivity to calcium-induced permeability transition pore opening even in the absence of BCAA or BCKA treatment [11]. The molecular basis of this observation remains unclear, but it may imply that branched-amino acid metabolism is functionally coupled with mitochondrial inner membrane permeability, which has a key role in mitochondrial calcium, respiration coupling, ROS, and cellular viability.

\section{Perspectives}

Branched-chain amino acids are important nutrient molecules with a potent signaling effect. Free BCAAs and their catabolic intermediates, BCKAs, are tightly maintained in animals by a highly regulated catabolic pathway. Defects in catabolism of BCAAs cause maple syrup urine disease, one of the most common metabolic disorders in the human population.

Although most clinical features of maple syrup urine disease are neurologic, our recent findings from cellular studies and animal models suggest that missing a key regulator in the catabolism of BCAAs also can cause a significant impairment in cardiac function (Fig. 4). The implication of this observation remains to be further established. However, a recent study based on metabolic profiling of peripheral blood has demonstrated a link between abnormal metabolism of BCAAs and coronary

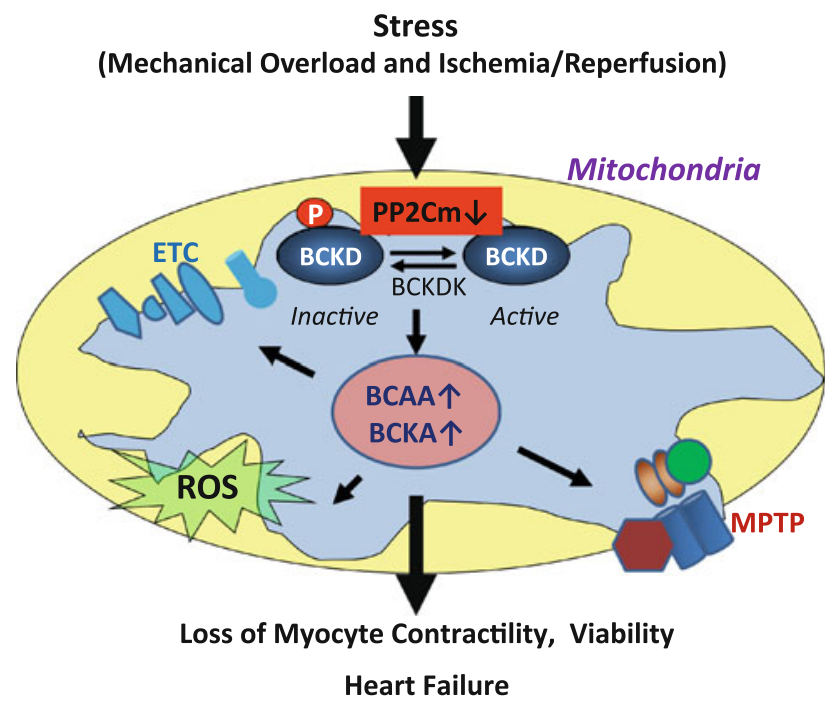

Fig. 4 Potential role played by catabolism of branched-chain amino acids (BCAA)/branched-chain alpha keto acids (BCKA) in heart failure. Schematic illustration showing the potential negative impact of an elevated BCAA/BCKA level on cardiac function via its regulation of mitochondrial electrophone transfer chain (ETC), reactive oxygen species (ROS), and mitochondrial permeability transition pore (mPTP) diseases [21]. As the complete molecular components of BCAA catabolic pathways are being discovered, better diagnosis will be available to identify patients with nonclassic, intermediate, or inducible maple syrup urine disease based on sequencing evidence. The insights learned from our study argue strongly for a better understanding of the role that the catabolism of BCAAs plays in the heart and its potential impact on cardiac pathology in addition to its damage to the central nervous system.

Acknowledgments This work was supported in part by National Institutes of Health grants HL70079, HL103205, HL098954, HL080111, and HL088640. Yibin Wang is an established investigator of the American Heart Association. Haipeng Sun is a recipient of a postdoctoral fellowship and Gang $\mathrm{Lu}$ a recipient of predoctoral fellowship awards from the Great Western Affiliate of American Heart Association.

Open Access This article is distributed under the terms of the Creative Commons Attribution Noncommercial License which permits any noncommercial use, distribution, and reproduction in any medium, provided the original author(s) and source are credited.

\section{References}

1. Amaral AU, Leipnitz G, Fernandes CG, Seminotti B, Schuck PF, Wajner M (2010) Alpha-ketoisocaproic acid and leucine provoke mitochondrial bioenergetic dysfunction in rat brain. Brain Res 1324:75-84

2. Avruch J, Long X, Ortiz-Vega S, Rapley J, Papageorgiou A, Dai $\mathrm{N}$ (2009) Amino acid regulation of TOR complex 1. Am J Physiol Endocrinol Metab 296:E592-E602

3. Baquet A, Lavoinne A, Hue L (1991) Comparison of the effects of various amino acids on glycogen synthesis, lipogenesis, and ketogenesis in isolated rat hepatocytes. Biochem J 273(Pt 1): 57-62

4. Chotechuang N, Azzout-Marniche D, Bos C, Chaumontet C, Gausseres N, Steiler T, Gaudichon C, Tome D (2009) mTOR, AMPK, and GCN2 coordinate the adaptation of hepatic energy metabolic pathways in response to protein intake in the rat. Am J Physiol Endocrinol Metab 297:E1313-E1323

5. Chuang DT (1998) Maple syrup urine disease: it has come a long way. J Pediatr 132:S17-S23

6. Contrusciere V, Paradisi S, Matteucci A, Malchiodi-Albedi F (2010) Branched-chain amino acids induce neurotoxicity in rat cortical cultures. Neurotox Res 17:392-398

7. Fernstrom JD (2005) Branched-chain amino acids and brain function. J Nutr 135:1539S-1546S

8. Harper AE, Miller RH, Block KP (1984) Branched-chain amino acid metabolism. Annu Rev Nutr 4:409-454

9. Harris RA, Joshi M, Jeoung NH (2004) Mechanisms responsible for regulation of branched-chain amino acid catabolism. Biochem Biophys Res Commun 313:391-396

10. Kaye CI, Accurso F, La Franchi S, Lane PA, Hope N, Sonya P, GB S, Michele AL (2006) Newborn screening fact sheets. Pediatrics 118:e934-e963

11. Lu G, Ren S, Korge P, Choi J, Dong Y, Weiss J, Koehler C, Chen JN, Wang Y (2007) A novel mitochondrial matrix serine/threonine protein phosphatase regulates the mitochondria permeability transition pore and is essential for cellular survival and development. Genes Dev 21:784-796 
12. Lu G, Sun H, She P, Youn JY, Warburton S, Ping P, Vondriska TM, Cai H, Lynch CJ, Wang Y (2009) Protein phosphatase $2 \mathrm{Cm}$ is a critical regulator of branched-chain amino acid catabolism in mice and cultured cells. J Clin Invest 119:1678-1687

13. Marc Rhoads J, Wu G (2009) Glutamine, arginine, and leucine signaling in the intestine. Amino Acids 37:111-122

14. Meijer AJ (2008) Amino acid regulation of autophagosome formation. Methods Mol Biol 445:89-109

15. Newgard CB, An J, Bain JR, Muehlbauer MJ, Stevens RD, Lien LF, Haqq AM, Shah SH, Arlotto M, Slentz CA, Rochon J, Gallup D, Ilkayeva O, Wenner BR, Yancy WS Jr, Eisenson H, Musante G, Surwit RS, Millington DS, Butler MD, Svetkey LP (2009) A branched-chain amino acid-related metabolic signature that differentiates obese and lean humans and contributes to insulin resistance. Cell Metab 9:311-326

16. Nishimura J, Masaki T, Arakawa M, Seike M, Yoshimatsu H (2010) Isoleucine prevents the accumulation of tissue triglycerides and upregulates the expression of PPARalpha and uncoupling protein in diet-induced obese mice. J Nutr 140:496-500

17. Potier M, Darcel N, Tome D (2009) Protein, amino acids, and the control of food intake. Curr Opin Clin Nutr Metab Care 12:54-58
18. Rennie MJ, Bohe J, Smith K, Wackerhage H, Greenhaff P (2006) Branched-chain amino acids as fuels and anabolic signals in human muscle. J Nutr 136:264S-268S

19. Saha AK, Xu XJ, Lawson E, Deoliveira R, Brandon AE, Kraegen EW, Ruderman NB (2010) Downregulation of AMPK accompanies leucine- and glucose-induced increases in protein synthesis and insulin resistance in rat skeletal muscle. Diabetes 59(10):2426-2434

20. Schadewaldt P, Wendel U (1997) Metabolism of branched-chain amino acids in maple syrup urine disease. Eur $\mathrm{J}$ Pediatr 156(Suppl 1):S62-S66

21. Shah SH, Bain JR, Muehlbauer MJ, Stevens RD, Crosslin DR, Haynes C, Dungan J, Newby LK, Hauser ER, Ginsburg GS, Newgard CB, Kraus WE (2010) Association of a peripheral blood metabolic profile with coronary artery disease and risk of subsequent cardiovascular events. Circ Cardiovasc Genet 3:207-214

22. Stipanuk MH (2007) Leucine and protein synthesis: mTOR and beyond. Nutr Rev 65:122-129

23. Vary TC, Lynch CJ (2007) Nutrient-signaling components controlling protein synthesis in striated muscle. J Nutr 137:1835-1843

24. Yudkoff M, Daikhin Y, Nissim I, Horyn O, Luhovyy B, Lazarow A (2005) Brain amino acid requirements and toxicity: the example of leucine. J Nutr 135:1531S-1538S 